\title{
Aiming to Satisfy the Demands of Catalyst Characterization and Spectroscopy
}

\author{
A. Howie* \\ *Cavendish Laboratory, University of Cambridge, Madingley Road, Cambridge CB3 0HE, UK.
}

When properly interfaced to complementary approaches, the various techniques of advanced TEM and SEM are now accorded a crucial role in the realistic characterization of heterogeneous catalysts as used in industry [1]. With the pioneering development of environmental ETEM in the past few years, enormous strides have been made in extending these powerful methods to follow catalytic processes in the electron microscope $[2,3]$. Notable successes have included the elucidation of the active sites for butane oxidation in vanadium phosphate (VPO) catalysts [4], increased understanding of metal-support interactions $[5,6]$, the role of the Ba promoter in $\mathrm{Ru}$ ammonia synthesis catalysts [7] and dynamic shape changes in the $\mathrm{Cu}$ particles used in methanol synthesis catalysts [8]. In combination with white-line EELS, changes of oxidation states in ceria-based automotive catalysts have been followed [9]. ETEM has also been extended to study hydrogenation and polymerization reactions in wet conditions and at the modest operating temperatures increasingly relevant environmentally [10]. Still better compromises between HREM and in-situ microscopy will be available with aberration-corrected lenses although the possibilities of higher beam current may be limited by specimen damage considerations.

The pressure gap between these experiments and typical catalyst operating conditions is still enormous but may not be a serious problem in cases where the effect can be reliably addressed by recalculating the rate constants using standard thermodynamic theory [11]. Even when there is for example a pressure-induced change in surface adsorbate structure it may still be possible to make a comparison between high pressure operation and low pressure experiments carried out at reduced temperatures [12]. Nevertheless it is encouraging to note that there has been some success in adapting well established, photon-based spectroscopy techniques of molecular chemistry to the extreme conditions of pressure and temperature which are generally used. Thus recent reviews of true in-situ spectroscopy methods have tended to concentrate on IR spectroscopy, Raman spectroscopy, UV-VIS spectroscopy, electron paramagnetic resonance, NMR, Mossbauer spectroscopy, X-ray diffraction and EXAFS [13, 14].

Microscopists will be swift to note that, although some of these techniques allow characterization of active sites on an average basis, none of them convey full spatially-resolved information. Their enviable sensitivity to significant molecular properties at surfaces argues strongly for incorporating these facilities in our equipment. This would have the added advantage of giving microscopists a foot on one of the bridges across the pressure gap and thus providing at least further means of comparison between ETEM observations and the actual situation in a typical reactor. Identification of active sites and reaction pathways has also been demonstrated by close correlation of microscopy data with DFT computations despite doubts about theoretical handling of excited states [15].

With its ready facility for atomic-level imaging and localized spectroscopy of electronic properties on clean single crystal surfaces, scanned probe microscopy (SPM) has largely displaced conventional electron microscopy in surface science though not yet in catalyst studies. A notable contribution here (closely combined with DFT computations, EXAFS and molecular beam studies) 
was the synthesis, characterization and testing of an Au-Ni steam reforming catalyst [16]. Copromoted active metallic sites on the edge of $\mathrm{MoS}_{2}$ particles which may be relevant in hydrodesulphurization catalysts have also been identified in STM observations, again in an increasingly natural partnership with DFT computations [17]. Unless these results prove to be an example of the so-called materials gap faced by SPM, they could indicate that the small patches of $\mathrm{MoS}_{2}$ seen in electron micrographs of these catalysts may be more significant than the more visibly striking, three-dimensional $\mathrm{MoS}_{2}$-coated particles also present [18]. The role of the SPM in catalyst studies will surely increase now that high pressure instruments with the capability of following dynamic events are available [19]. It may not rival the TEM in chemical analysis but can study the electronic structure of surface sites and recognize individual small molecules on surfaces through their characteristic vibration signatures in tunneling spectroscopy [20]. There are also several options for using the SPM to confer high spatial resolution on various photon spectroscopies even under high pressure conditions. Thus in photoemission spectroscopy, excited electrons with insufficient energy to escape can be collected if they tunnel into an STM tip scanned close to the surface [21]. Through apertureless near-field methods, the SPM may also be readily compatible with various optical spectroscopies mentioned above, thus combining its high spatial resolution with the spectral precision, tuneability of excitation and other advantages of optical methods $[22,23]$. The influence of the tip on the molecules cannot be ignored however.

References

[1] J. Liu, Microsc. Microanal. 10 (2004) 55.

[2] P.L. Gai, Cat. Rev. Sci Eng. 34 (1992) 1.

[3] P.L. Gai and E.D. Boyes, Electron Microscopy in Heterogeneous Catalysis, Inst.of Physics, Bristol and Philadelphia, 2003.

[4] P.L. Gai and K. Kourtakis, Science 267 (1995) 661.

[5] P.L. Gai, Adv. Materials 10 (1998) 1259.

[6] P.Li et al., Microsc. Microanal. 10 (Suppl 2) (2004) 458.

[7] T.W. Hansen et al., Science 294 (2001) 1508.

[8] P.L. Hansen et al., Science 295 (2002) 2053.

[9] R. Sharma et al., Phil. Mag. 84 (2004) 2731.

[10] P.L. Gai, Microsc. Microanal. 8 (2002) 21.

[11] P. Stoltze and J.K. Norskov, Phys. Rev. Lett. 55 (1985) 852.

[12] L. Osterlund et al. Phys. Rev. Lett. 86 (2001) 460.

[13] B.M. Weckhuysen, Chemical Communications (2002) 97.

[14] J. Haw, In-Situ Spectroscopy in Heterogeneous Catalysis, Wiley VCH, Weinheim, 2002.

[15] K. Honkala et al., Science 307 (2005) 555.

[16] F. Besenbacher et al., Science 279 (1998) 1913.

[17] S. Helvig et al., Phys. Rev. Lett. 84 (2000) 951.

[18] J.V. Sandars, Chemica Scripta 14 (1978) 141.

[19] E. Laegsgaard et al., Rev. Sci. Instrum. 72 (2001) 3537.

[20] L.J. Lauhon and W. Ho, Phys. Rev. B 60 (1999) R8525.

[21] S. Gray, Electron Spectr. And Related Phenom. 109 (2000) 183.

[22] Y. Martin et al., Appl. Phys. Lett. 68 (1996) 2475.

[23] H. Watanabe et al., Phys. Rev. B 69 (2004) 155418.

I thank Dupont for conference travel support. 